\title{
PSO Watermarking Model for Multimodal Biometric System
}

\author{
S. Anu H Nair \\ Assistant Professor \\ Department of Computer Science \& Engg. \\ Annamalai University.
}

\author{
P. Aruna, $\mathrm{PhD}$ \\ Professor \\ Department of Computer Science \& Engg. \\ Annamalai University.
}

\begin{abstract}
Multimodal Biometric Watermarking System using multiple sources of information for establishing the individuality has been widely recognized, computational models for multimodal biometrics recognition have only recently got attention. In this paper multimodal biometric images such as fingerprint, palmprint, and iris are extracted individually and are fused together using Average, Minimum and Maximum fusion mechanism. The fused template is then watermarked using the PSO watermarking system. The biometric features used here are fingerprint, iris and palmprint. The image quality is measured by using various metrics such as Peak Signal Noise ratio(PSNR), Normalized Absolute Error(NAE) and Normalized Cross Correlation(NCC). CASIA database is chosen for the biometric images. All the images are 8 bit graylevel JPEG image with the resolution of $320 * 280$.
\end{abstract}

\section{Keywords}

Biometric, watermarking, fusion, Peak Signal Noise ratio (PSNR), Normalized Absolute Error(NAE) and Normalized Cross Correlation(NCC).

\section{INTRODUCTION}

Biometrics refers to the automatic recognition of individuals based on their physiological and behavioral characteristics. Physiological biometrics (fingerprint, iris, retina, hand geometry, face, etc.) use measurements from the human body. Behavioral biometrics (signature, keystrokes, voice, etc.) use dynamics measurements based on human actions. Universality, uniqueness, permanence, collectability, acceptability, resistance to circumvention, the performances are the characteristics of biometric features. The physical characteristics taken are, Fingerprint: A fingerprint is the pattern of ridges and valleys on the surface of a fingertip. It has been empirically determined that the fingerprints of identical twins are the feature values typically correspond to the position and orientation of certain critical points known as minutiae points. Iris: The iris is the annular region of the eye bounded by the pupil and the sclera (white of the eye) on either side. The complex iris texture carries very distinctive information useful for personal recognition of high accuracy and speed. Each Iris is believed to be distinctive. It is possible to detect artificial irises (contact lenses). Palm prints: The palms of the human hands contain a pattern of ridges and valleys much like the fingerprints. Human palms also contain additional distinctive features such as principal lines and wrinkles that can. It is easy to be captured even with a lower resolution scanner.

Unimodal biometric systems rely on a single source of information such as a single iris or fingerprint or palmprint for authentication. Unfortunately these systems have to deal with some of the following inevitable problems. Noisy data, Nonuniversality, Intra-class variations, Spoof attack. It has been observed that some of the limitations of unimodal biometric systems can be addressed by deploying multimodal biometric systems that essentially integrate the evidence presented by multiple sources of information such as iris, fingerprints and palmprints. Such systems are less vulnerable to spoof attacks as it would be difficult for an imposter to simultaneously spoof multiple biometric traits of a genuine user. Due to sufficient population coverage, these systems are able to address the problem of non-universality.

Multi-biometric: The term multi-biometrics denotes the fusion of different types of information (e.g., fingerprint and face of the same person, or fingerprints from two different fingers of a person). Multi-biometrics has addressed some issue related to unimodal such as, (a) Non-universality or insufficient population coverage (reduce failure to enroll rate which increases population coverage). (b) It becomes increasingly difficult for an impostor to spoof multiple biometric traits of a legitimately enrolled individual. (c) Multi-biometric systems also effectively address the problem of noisy data (illness affecting voice, scar affecting fingerprint.

Classification in multi-biometric systems are done by fusing information from different biometric modalities. Information fusion can be done at different levels, broadly, divided into feature-level, score-level and rank/decision-level fusion. Due to preservation of raw information, feature-level fusion can be more discriminative than score or decision-level fusion. But, feature-level fusion methods are being explored in the biometric community only recently. This is because of the differences in features extracted from different sensors in terms of type and dimensions. Often features have large dimensions, and fusion becomes difficult at the feature level. The prevalent method is feature concatenation, which has been used for different multi-biometric settings. However, for high dimensional feature vectors, simple feature concatenation may be inefficient and non-robust. In recent years, a theory of Sparse Representation (SR) has emerged as powerful tools for efficient processing of data in nontraditional ways.

A digital watermark is a pattern of bits inserted into a digital file - image, audio or video. Such messages usually carry copyright information of the file. Digital watermarking takes its name from watermarking of paper or money. The types of watermarking are division based on human perception. This is sub-divided into visible watermarks and invisible watermarks. Visible Watermarks: These watermarks can be seen clearly by the viewer and can also identify the logo or the owner. Visible watermarking technique changes the original signal. The watermarked signal is different from the original signal. 
Spreading the watermark throughout the image is a best option, but the quality of the image is degraded which prevents the image from being used in medical applications. Invisible Watermarks: These watermarks cannot be seen by the viewer. The output signal does not change much when compared to the original signal. Invisible watermarking is more robust to signal processing attacks when compared to visible watermarking. Based on application watermarks are sub-divided into fragile, semi-fragile and robust watermarks. Various types of watermarking algorithm are done successfully to perform watermarking.

In that respect are different optimization methods and algorithms that can be grouped into deterministic and stochastic.tochastic.. Deterministic techniques depend on the mathematical nature of the problem. Weaknesses of this technique are dependent on the gradient, local optimums, and inefficiency in large-scale search space. Stochastic techniques are seen to be more user friendly because they do not depend on the mathematical attributes of a given function and are therefore more appropriate for determining the global optimal solutions for whatever type of objective function. As many real-world optimization problems become increasingly complex, using stochastic methods is inevitable.

\section{LITERATURE REVIEW}

[1] proposed a fingerprint classification method where types of singular points and the number of each type of point are chosen as features. [2] designed an orientation diffusion model for fingerprint extraction where corepoints and ridgeline flow are used. [3] created a novel minutiae based fingerprint matching system which creates a feature vector template from the extracted core points and ridges. [4] modeled a palmprint based recognition system which uses texture and dominant orientation pixels as features. [5] identified a palmprint recognition method which uses blanket dimension for extracting image texture information. [6] presented a typical palmprint identification system which constructed a pattern from the orientation and response features. [7] designed a new palmprint matching system based on the extraction of feature points identified by the intersection of creases and lines. [8] proposed a competent representation method which can be used for classification. [9] created a model that fused voice and iris biometric features. This model acted as a new representation of existing biometric data. [10] proposed user specific and selective fusion strategy for an enrolled user. [11] identified a new geometrical feature Width Centroid Contour Distance for finger geometry biometric. [12] developed a face and ear biometric system which uses a feature weighing scheme called Sparse Coding error ratio. [13]proposed the fusion method based on a compressive sensing theory which contains over complete dictionary, an algorithm for sparse vector approximation and fusion rule. [14] identified the feature extraction techniques for three modalities viz. fingerprint, iris and face. The extracted data is stored as a template which can be fused using density based score level fusion. [15] proposed a PSO watermarking algorithm where the optimized particle are identified.The fitness function used here is universal image quality index which is used to identify the best particle. Then the watermark is embedded in the best identified position.

\section{PROPOSED WORK}

The proposed work describes the feature extraction of multimodal biometric images such as, fingerprint, palmprint, and iris. The extracted information is fused together using average,minimum and maximum fusion. The best fused template is then watermarked into a host image using PSO algorithm and extracted further.
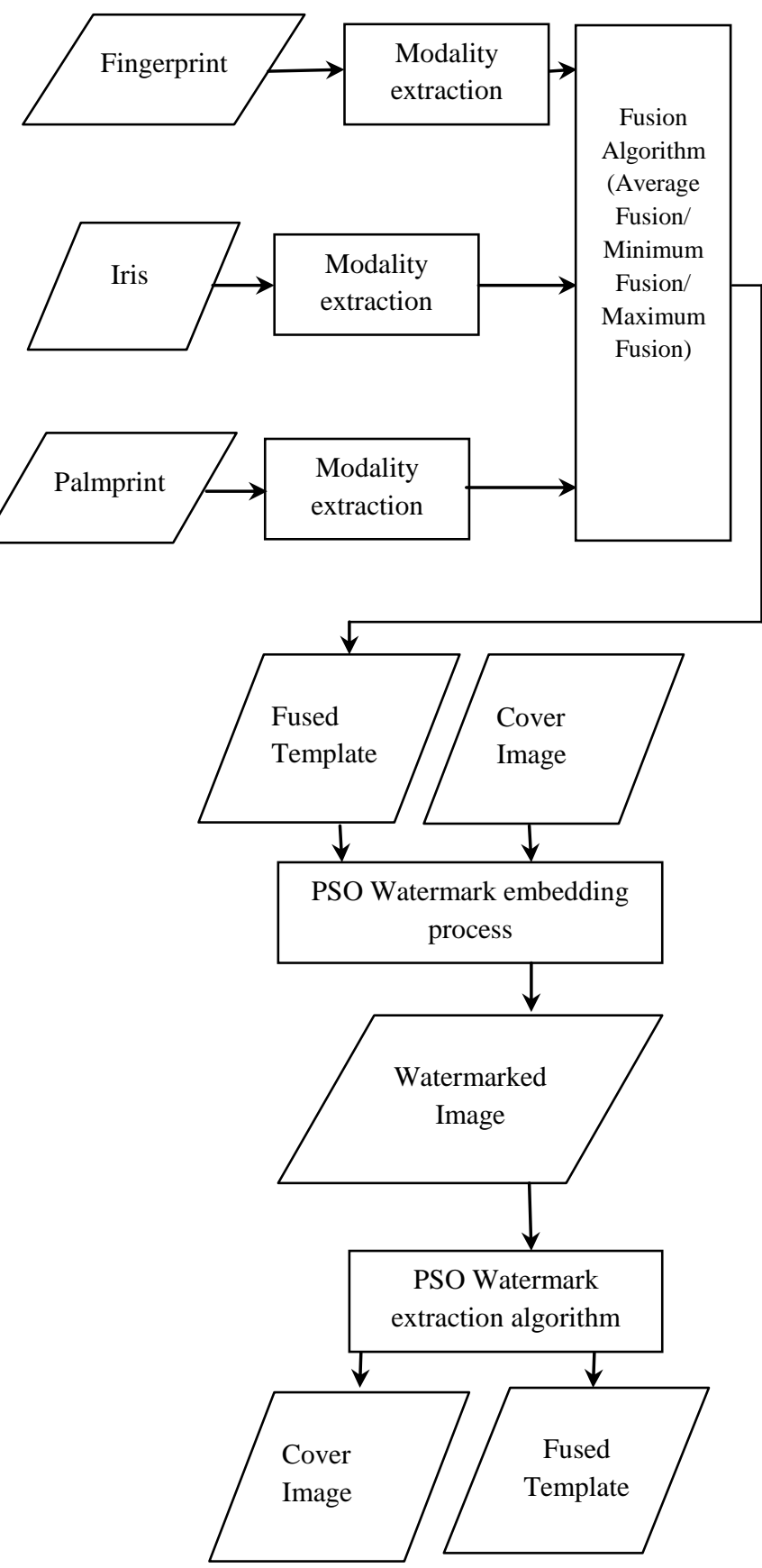

Fig.1 Flow diagram of the proposed work 


\subsection{Modality extraction from a Fingerprint image}

The fingerprint image is fed as the input. The contrast of the grayscale image is enhanced by using the Adaptive histogram equalization technique (CLAHE). Orientation process is used to find the direction of the ridges in the fingerprint image. This can be achieved by using the SOBEL filter to detect the edges of the image.ROI selection is used to give maximum magnitude of convolution in the region of core point. This fingerprint masking is used to select the region where the fingerprint images are present. The width of a fingerprint pattern carries no useful information for our immediate purpose and is eliminated by a thinning operation. Thinning algorithms reduce connected patterns to a width of a single pixel while maintaining their topology. The thinning is performed using Morphological operation. Once this is done, the feature of the fingerprint is successfully extracted.

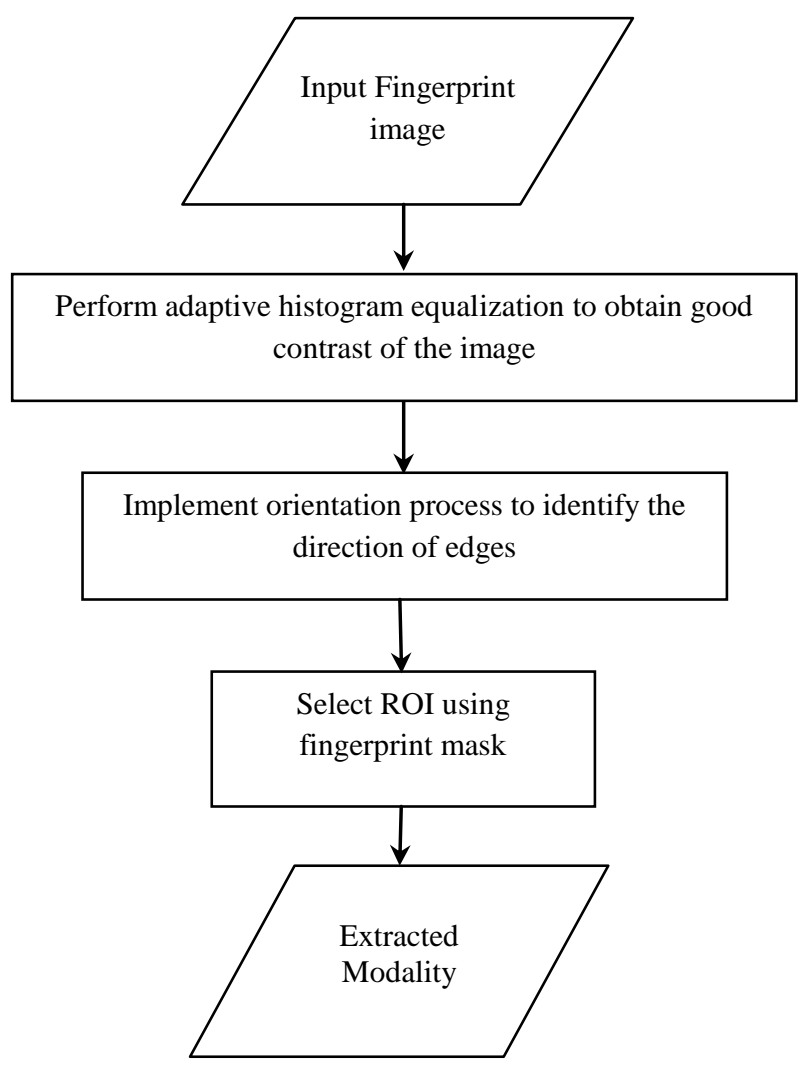

Fig.2 Flow diagram of fingerprint modality extraction process

\subsection{Modality extraction from a Iris image}

The iris image is fed as the input. Morphological operation is performed on the input image to erode or dilate pixels. The Fourier transform is applied to the image to filter the image based on frequency. Then edge detection process using sobel filter is performed. The Hough Transform is applied to the filtered image to find the straight lines (functions) hidden in larger amounts of other data. For detecting lines in images, the image is first binarised using some form of thresholding and then the positive instances catalogued. Adaptive histogram technique is applied finally to enhance the contrast of the image. Once this is done, the feature of the iris is successfully extracted.

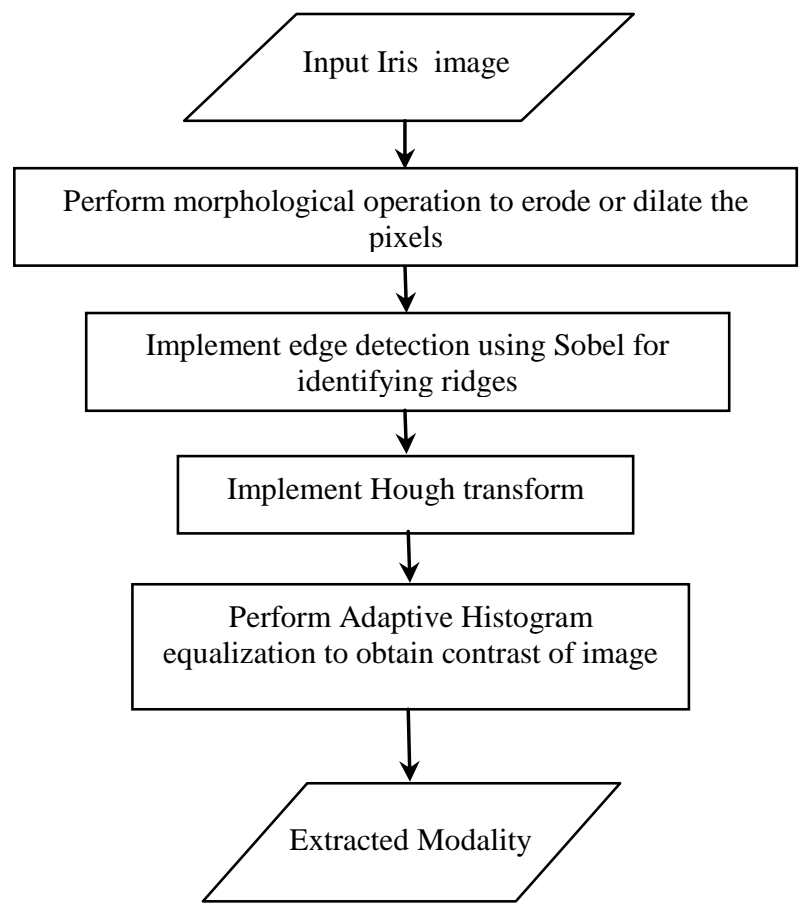

Fig.3 Flow diagram of iris modality extraction process

\subsection{Modality extraction from a Palmprint image}

The palmprint image is fed as the input. The contrast of the grayscale image is enhanced by using the Adaptive histogram equalization technique (CLAHE). Diffusion filter is used to remove noise from an image by modifying the image via a partial differential equation (PDE). Edge detection is performed using sobel filter to identifying the ridges. Thinning algorithms reduce connected patterns to a width of a single pixel while maintaining their topology. The thinning is performed using Morphological operation. Oncethis is done, the feature of the Palmprint is successfully extracted.

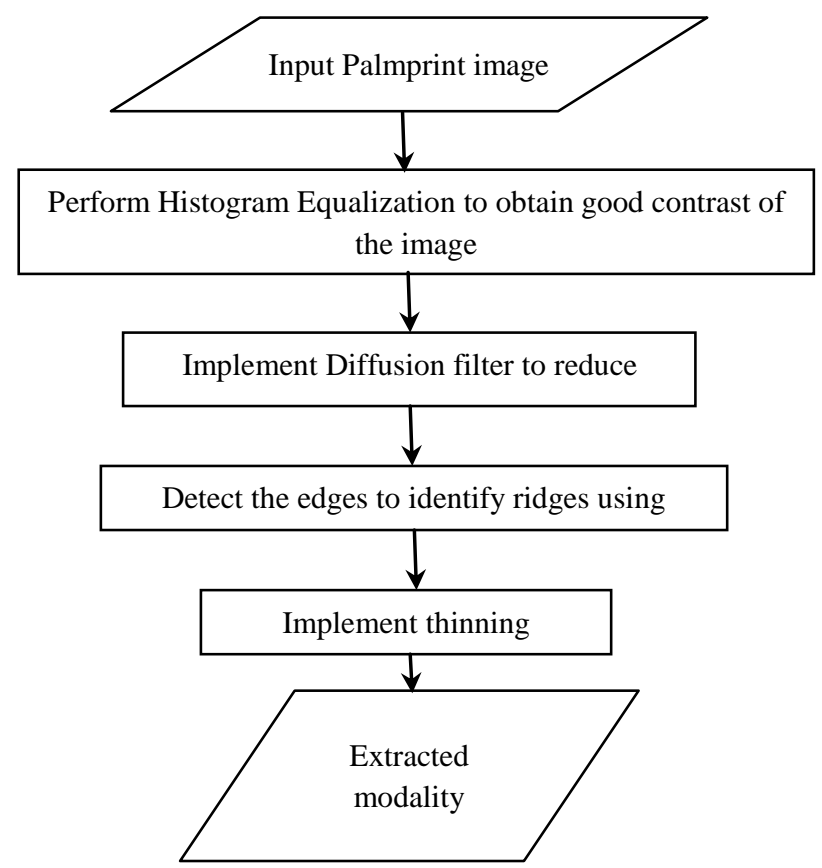

Fig.4 Flow diagram of palmprint modality extraction process 


\section{FUSION MECHANISM}

The extracted modalities are fused together using the fusion methods Average, minimum and maximum fusion methods.Here the fingerprint extracted modality and iris extracted modality are given as input and the fusion methods are implemented.The output fused template is further subjected to the same fusion methods. Thus the fused template is obtained as the output. The flow diagram is shown in Figure 5

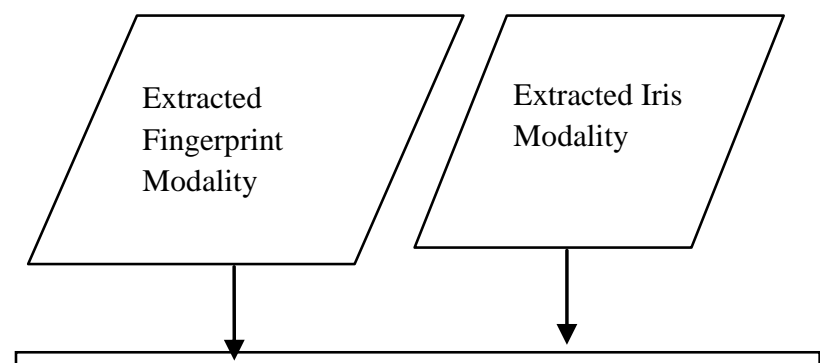

Various types of fusion mechanism such as Average Fusion/Maximum Fusion/ MinimumFusion

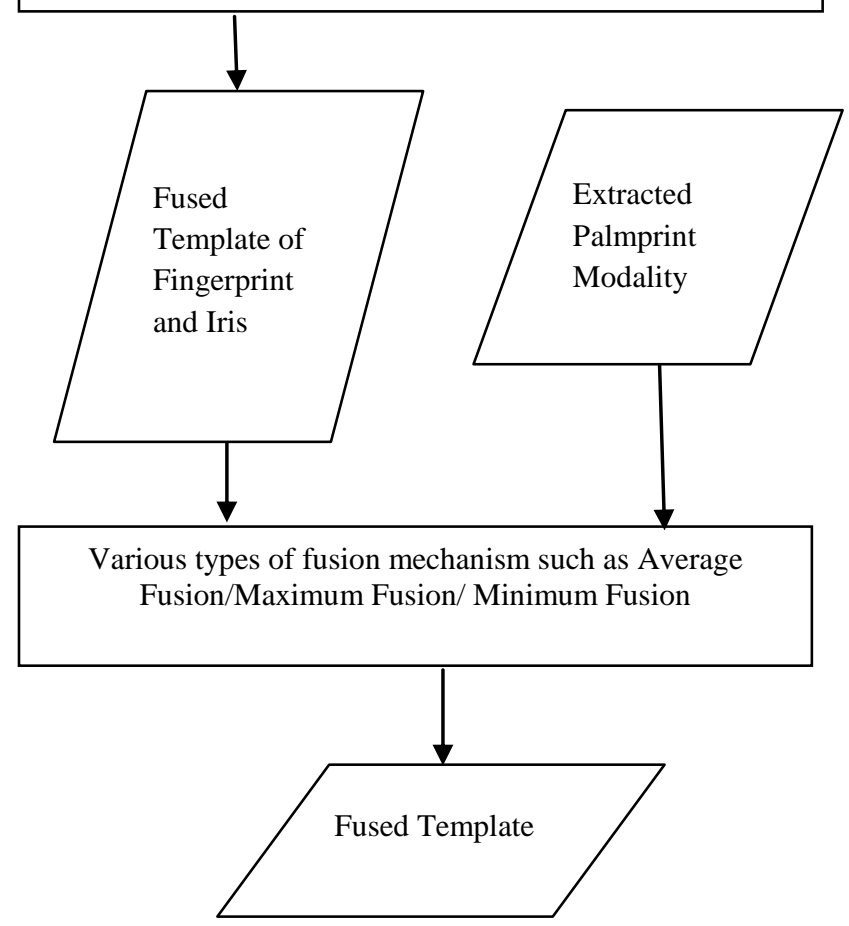

Fig.5 Flow diagram of fusion of 3 extracted modalities

\subsection{Average Fusion method}

The values of the pixel $(i, j)$ of each image $\mathrm{X}$ and $\mathrm{Y}$ are taken and added. This sum is then divided by 2 to obtain the average. The average value is assigned to the corresponding pixel of the output image. This is repeated for all pixel values.[16]

$$
K(i, j)=(X(i, j)+Y(i, j)) / 2
$$

Here the fingerprint and iris extracted modalities are taken as input and the fusion method is implemented. Then the fused template and the palmprint extracted modality are taken as input which produces the fusion of three modalities as output.

\subsubsection{Output for average fusion}

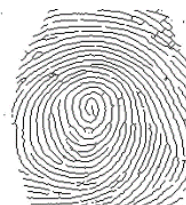

(a)

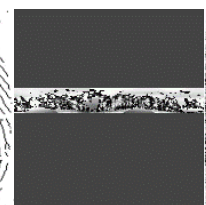

(b)

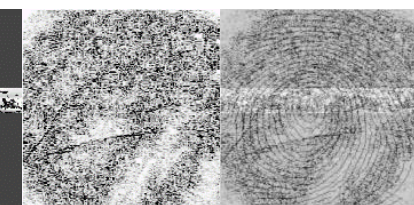

(c) (d)
Fig. 6.The extracted modaliteies of (a)fingerprint,(b)iris and (c) palmprint and their average fused template(d)

\subsection{Minimum/Maximum fusion}

For every corresponding pixel in the input images, the pixel with maximum/minimum intensity is selected This is put in as the resultant pixel of the fused image.

\subsubsection{Output for minimum fusion}

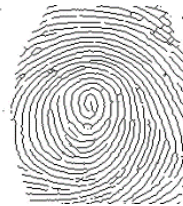

(a)

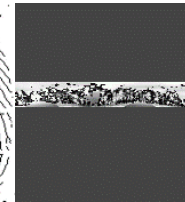

(b)

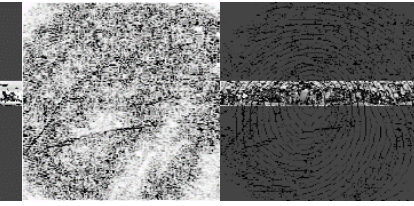

(c)

(d)
Fig. 7. The extracted modalities of (a) fingerprint, (b) iris and (c) palmprint and their minimum fused template(d)

\subsubsection{Output for maximum fusion}

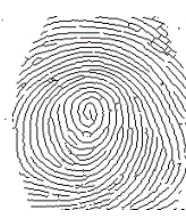

(a)

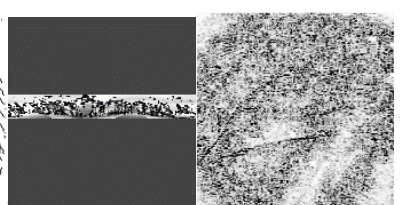

(b)

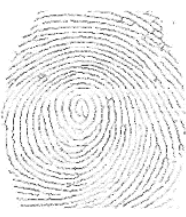

(d)
Fig. 8. The extracted modaliteies of (a)fingerprint,(b)iris and (c) palmprint and their maximum fused template(d)

\section{PERFORMANCE METRICS FOR ANALYZING FUSED TEMPLATES}

\subsection{Xydeas and Petrovic Metric- $Q^{\mathrm{AB} / \mathrm{F}}$}

Mathematically $\mathrm{Q}^{\mathrm{AB} / \mathrm{F}}$ is defined as

$$
Q^{A B / F}=\frac{\sum_{m=1}^{M} \sum_{n=1}^{N}\left[Q_{m, n}^{A F} W_{m, n}^{A}+Q_{m, n}^{B F} W_{m, n}^{B}\right]}{\sum_{m=1}^{M} \sum_{n=1}^{N}\left[W_{m, n}^{A}+W_{m, n}^{B}\right]}
$$


where $\mathrm{A}, \mathrm{B}$ and $\mathrm{F}$ represent the input and fused images respectively. The definition of $Q_{m, n}^{A F}$ and $Q_{m, n}^{B F}$ are the same and given as

$Q_{m, n}^{A F}=Q_{g}^{A F} m, n+Q_{\alpha}^{A F} m, n$

where $Q_{g}^{* F}, Q_{\alpha}^{* F}$ are the edge and orientation values at location $(\mathrm{m}, \mathrm{n})$ of images $\mathrm{A}$ and $\mathrm{B}$. The dynamic range for $Q^{A B / F}$ is [0,1] and it should be close to 1 for better fusion.

\subsection{Visual Information Fidelity(VIF)}

VIF first decomposes the image into several subbands and parses each sub-band into blocks. This measures by computing mutual information in each block and in each subband.Finally the image quality is measured by integrating visual information for all blocks and subbands.

VIF=Distorted Image information/Reference Image Information

\subsection{Fusion Mutual Information}

This measures the degree of dependence of 2 images. Fused Mutual Information(FMI) is defined as

$$
F M I=M I_{I_{1} I_{f}}+M I_{I_{2} I_{f}}
$$

where

$$
\begin{aligned}
& M I_{I_{1} I_{f}}=\sum_{i=1}^{M} \sum_{j=1}^{N} h I_{I_{1} I_{f}}(i, j) \log _{2}\left(\frac{h I_{I_{1} I_{f}}}{h I_{1}(i, j) h I_{f}(i, j)}\right) \\
& M I_{I_{2} I_{f}}=\sum_{i=1}^{M} \sum_{j=1}^{N} h I_{I_{2} I_{f}}(i, j) \log _{2}\left(\frac{h I_{I_{2} I_{f}}}{h I_{2}(i, j) h I_{f}(i, j)}\right)
\end{aligned}
$$

A large measure of Fusion Mutual Information implies better quality.

\subsection{Entropy}

Entropy is defined as amount of information contained in a signal. The entropy of the image can be evaluated as

$$
H=-\sum P(i) * \log _{2}\left(P\left(d_{i}\right)\right)
$$

where $\mathrm{G}$ is the number of possible gray levels, $P\left(d_{i}\right)$ is probability of occurrence of a particular gray level $d_{i}$. If entropy of fused image is higher than parent image then it indicates that the fused image contains more information

\subsection{Average Gradient}

The Average Gradient is applied to measure the detailed information in the images.

\begin{tabular}{|c|c|c|c|c|c|}
\hline $\begin{array}{l}\text { Metrics } \\
\text { Fusion } \\
\text { method }\end{array}$ & Qabf & VIF & MI & $\begin{array}{c}\text { Average } \\
\text { Gradient }\end{array}$ & Entropy \\
\hline $\begin{array}{l}\text { Average } \\
\text { Fusion }\end{array}$ & 0.35 & 0.22 & 3.08 & 15.15 & 6.76 \\
\hline $\begin{array}{l}\text { Minimum } \\
\text { Fusion }\end{array}$ & 0.22 & 0.10 & 1.88 & 9.61 & 4.02 \\
\hline $\begin{array}{l}\text { Maximum } \\
\text { Fusion }\end{array}$ & 0.21 & 0.09 & 1.82 & 8.89 & 3.97 \\
\hline
\end{tabular}

$$
g=\frac{1}{(M-1) \cdot(N-1)} \sum_{x=1}^{M-1} \sum_{y=1}^{N-1} \sqrt{\left(\frac{\partial f(x, y)}{\partial x}\right)^{2}+\left(\frac{\partial f(x, y)}{\partial y}\right)^{2}}
$$

Table-I. Performance analysis of above fused templates

\section{ANALYSIS OF VARIOUS FUSION METHODS}

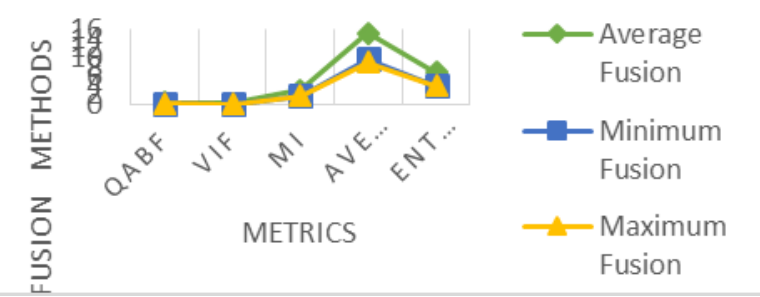

Fig.9 Analysis of various fusion methods

From the above results average fusion method provides better results than other fusion methods. Therefore the average fused template is fed as input to the PSO watermarking system along with cover image. Table I and Figure 9 depicts the same.

\section{PARTICLE SWARM OPTIMIZATION (PSO) WATERMARKING}

All the particles have fitness values which are calculated by the objective function to be optimized by the PSO algorithm and have velocities which direct the movement of the particles.[15] Let $x i(t)$ denote the position of particle $i$ in the search space at time step t. The position of the particle is changed to $x_{i}(t+1)$ by adding a velocity $v_{i}(t+1)$ to the current position, i.e.

$$
x_{i j}(t+1)=x_{i j}(t)+v_{i j}(t+1)
$$

The initial population is created in such a way that the particles are distributed randomly over the search space. It is the velocity vector that drives the optimization process, and reflects both the experiential knowledge of the particle and socially exchanged information from the particle's neighborhood. The experimental knowledge of a particle is generally referred to as the cognitive component, which is proportional to the distance of the particle from its own best position called pbest found since the first time step. The socially exchanged information is referred to as the social component of the velocity equation. The social component of the particle velocity update reflects information obtained from all the particles in the swarm. In this case, the social information is the best position found by the swarm, referred to as gbest. At each iteration, each particle is updated with respect to these two values pbest and gbest.

The rate of change of position of particle is defined as

$\mathrm{v}_{\mathrm{ij}}(t+1)=w v_{i j}(t)+c_{l} \operatorname{random}_{1}[$ pbest-xij $(\mathrm{t})]+\mathrm{c}_{2}$ 
where $\mathrm{c} 1$ and $\mathrm{c} 2$ are constants and random1 and random2 ranges between 0 and 1 . In PSO every particle has a velocity which is to be determined. The best particle is identified by the evaluation of the fitness function.

The algorithm is as follows:

- Initialize the position and velocity of the particle.

- At each iteration the velocities of all particles are updated

- Using eq the positions of particles are updated

- Update the pbest and gbest by

$$
\begin{aligned}
\text { pbest } & =p_{k} \text { if } f\left(p_{k}\right)>\text { pbest } \\
\text { gbest } & =g_{k} \text { if } f\left(g_{k}\right)>\text { gbest }
\end{aligned}
$$

After the optimal block is identified the fused template is watermarked into the block.This results in the watermarked image. The reverse process is applied for extraction of the fused template from the watermarked image.

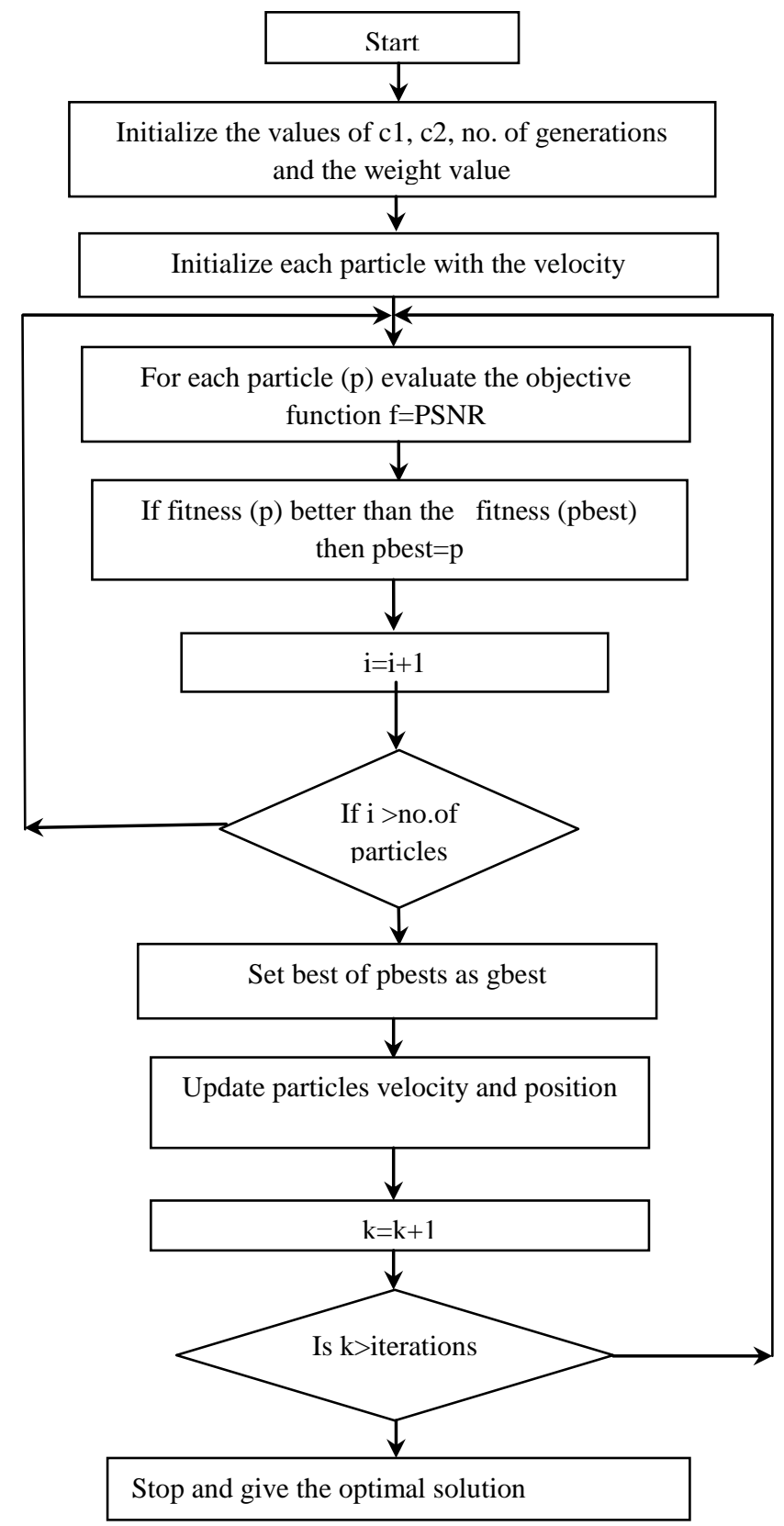

Fig.10 Particle Swarm optimization algorithm

\section{PERFORMANCE METRICS}

\subsection{Peak signal-to-noise ratio (PSNR)}

Peak Signal Noise Ratio represents the measure of peak error. Inorder to compute PSNR the mean squared error (MSE) is first calculated.

$$
M S E=\frac{1}{M N} \sum_{i=0}^{M-1} \sum_{j=0}^{N-1}[I(i, j)-K(i, j)]^{2}
$$

where I, $\mathrm{K}$ are images, $\mathrm{M}, \mathrm{N}$ are rows and columns of images.

$$
P S N R=10 \log _{10} \frac{255^{2}}{M S E}
$$

An increase in PSNR implies high quality image.

\subsection{Normalized absolute error(NAE)}

Normalized absolute error is the total normalized error values between the cover image and the watermarked images. The large value of Normalized Absolute Error (NAE) means that the image is of poor quality. NAE is defined as follows:

$$
N A E=\sum_{j=1}^{M} \sum_{k=1}^{N}\left|x_{j, k}-x_{j, k^{\prime}}\right| / \sum_{j=1}^{M} \sum_{k=1}^{N}\left|x_{j, k}\right|
$$

\subsection{Normalized cross correlation (NCC)}

The metric is calculated as the ratio between the net sum of the multiplication of the corresponding pixel densities of the watermarked image and the cover image and the net sum of the squared values of the pixel densities of the cover image.

$$
N C C=\sum_{i=1}^{M} \sum_{j=1}^{N} \frac{\left(A_{i, j}{ }^{*} B_{i, j}\right)}{\sum_{i=1}^{M} \sum_{j=1}^{N}\left(A_{i, j}\right)^{2}}
$$

where $\mathrm{A}$ and $\mathrm{B}$ are images, $\mathrm{M}$ and $\mathrm{N}$ are rows and columns of images. The Normalized Cross Correlation value would be ideally 1 if both images are identical.

\section{EXPERIMENTAL RESULTS AND PERFORMANCE ANALYSIS}

The PSO watermarking system was experimented with different cover images and their performance analysis was performed using different quality metrics PSNR, NCC and NAE. Table- II demonstrates the same.

Table-II. Performance Analysis of different watermarked images using a PSO watermarking system

\begin{tabular}{|c|c|c|c|}
\hline $\begin{array}{c}\text { STANDARD } \\
\text { IMAGES }\end{array}$ & PSNR(dB) & NCC & NAE \\
\hline BOAT & 33.87 & 0.52 & 0.082 \\
\hline LENA & 34.112 & 0.54 & 0.085 \\
\hline COUPLE & 34.29 & 0.56 & 0.086 \\
\hline BABOON & 36.45 & 0.58 & 0.089 \\
\hline
\end{tabular}


Table-III. Sample output obtained by applying PSO watermarking system

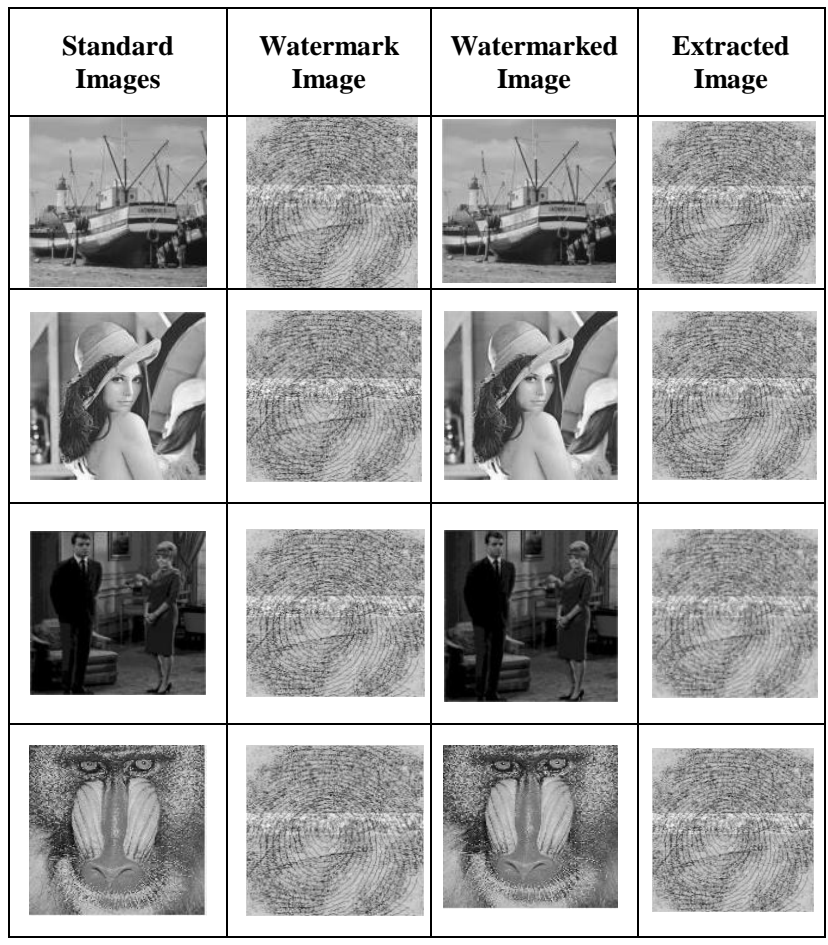

\section{CONCLUSION}

In this paper a novel feature level fusion algorithm for multimodal biometric features is proposed. Each biometric feature is individually extracted and the obtained modalities were fused together using average ,minimum and maximum fusion methods. Then the best fused template is identified by analyzing their quality using different performance metrics.This fused template has been watermarked in different cover images. Various metrics such as PSNR, NAE and NCC are used to measure the image quality. CASIA database is chosen for the biometric images. All the images are 8 bit graylevel JPEG image with the resolution of $320 * 280$.

\section{REFERENCES}

[1] Jing-Ming Guo, Yun-Fu Liu, Jla-Yu Chang, Jiann-Der Lee, "Fingerprint classification based on decision tree from singular points and orientation field ", Elsevier, 2014.

[2] Kai Cao, Liaojun Pang, Jimin Liang, Jie Tia, "Fingerprint classification by a hierarchical classifier", Elsevier, 2013.
[3] Ayman Mohammad Bahaa-Eldin, "A medium resolution fingerprint matching system”, Elsevier, 2013.

[4] Kamlesh Tiwari, Devendra K. Arya, G.S. Badrinath, Phalguni Gupta, "Designing palmprint based recognition system using local structure tensor and force field transformation for human identification", Elsevier, 2013.

[5] Xiumei Guo, Weidong Zhou, Yu Wang, "Palmprint recognition algorithm with horizontally expanded blanket dimension", Elsevier, 2014.

[6] Feng Yue, Wangmeng Zuo, "Consistency analysis on orientation features for fast and accurate palmprint identification”, Elsevier, 2013.

[7] O. Nibouche, J. Jiang, "Palmprint matching using feature points and SVD factorisation", Elsevier, 2013.

[8] Jing Li, Jian Cao, Kaixuan Lu, "Improve the two-phase test samples representation method for palmprint recognition", Elsevier, 2013.

[9] Anne M.P. Canuto, Fernando Pintro, João C. XavierJunio, "Investigating fusion approaches in multibiometric cancellable recognition”, Elsevier, 2013.

[10] Norman Poh, Arun Ross, Weifeng Lee, Josef Kittle, “A user-specific and selective multimodal biometric fusion strategy by ranking subjects", Elsevier, 2013.

[11] Mohd Shahrimie Mohd Asaari, Shahrel A. Suandi, Bakhtiar Affendi Rosdi, "Fusion of Band Limited Phase Only Correlation and Width Centroid Contour Distance for finger based biometrics", Elsevier, 2014.

[12] Zengxi Huang, Yiguang Liu, Chunguang Li, Menglong Yang, Liping Chen, "A robust face and ear based multimodal biometric system using sparse representation", Elsevier, 2013.

[13] Meng Ding, Li Wei, Bangfeng Wang ,"Research on fusion method for infrared and visible images via compressive sensing”, Elsevier, 2013.

[14] Mr.J.Aravinth, Dr.S.Valarmathy, “A Novel Feature Extraction Techniques for Multimodal Score Fusion Using Density Based Gaussian Mixture Model Approach", IJETAE, Jan 2012.

[15] K.Kuppusamy,K.Thamodaran,"Optimized Image Watermarking Scheme Based on PSO”, Procedia Engineering,Elsevier 2012.

[16] Sunil Kumar Panjeta, Deepak Sharma, "A Survey on image fusion techniques used to improve image quality",International Journal of Engineering and research,2012. 